\title{
Evaluation of ablation differences in air and water for hard tissues using full-field optical coherence microscopy
}

\author{
LÜ Tao ${ }^{1,2}$, XIAO Qing ${ }^{3}$, LI ZhengJia ${ }^{2} \&$ FU Ling ${ }^{3 *}$ \\ ${ }^{1}$ School of Mathematics and Physics, China University of Geosciences, Wuhan 430074, China; \\ ${ }^{2}$ Wuhan National Laboratory for Optoelectronics, College of Optoelectronics Science and Engineering, Huazhong University of Science and \\ Technology, Wuhan 430074, China; \\ ${ }^{3}$ Britton Chance Center for Biomedical Photonics, Wuhan National Laboratory for Optoelectrics, Huazhong University of Science and Technology, \\ Wuhan 430074, China
}

Received September 21, 2011; accepted October 27, 2011; published online January 2, 2012

\begin{abstract}
To clarify the role of a natural or artificial liquid environment in the free-running infrared pulsed laser ablation of hard biological tissues, two- and three-dimensional morphologies of laser-induced craters must be quantitatively measured to distinguish ablation differences in air and in water. Full-field optical coherence microscopy is introduced, which has non-contact, non-destructive, non-preprocessing and higher-resolution advantages. Experimental results indicate that the ablation performances in air and in water are comparable for few laser pulses, but the ablation difference becomes obvious for more laser pulses. Optical coherence microscopy is more feasible than conventional means for the morphological measurement of craters.
\end{abstract}

ablation of hard tissue, optical coherence microscopy, holmium laser pulse

Citation: Lü T, Xiao Q, Li Z J, et al. Evaluation of ablation differences in air and water for hard tissues using full-field optical coherence microscopy. Chin Sci Bull, 2012, 57: 833-837, doi: 10.1007/s11434-011-4889-x

Enhancement of material ablation by an artificially deposited liquid film or in a natural liquid environment during short-pulse ( $\tau<1 \mu \mathrm{s}$, femtosecond [1] and nanosecond [2] domains) or long-pulse $(\tau>1 \mu \mathrm{s})$ [3-5] laser ablation has been investigated in recent years. In clinical applications, ablation in a liquid environment (e.g. water, blood, saline, or a mixture of them) (IALE) is often encountered when delivering laser radiation through optical fibers for medical applications inside the human body [6]; for example, in arthroscopic surgery [7], orthopedic procedures [8], dentistry [3], osteotomy [4], angioma treatment [9], and urology [10]. However, unlike the case for short-pulse laser ablation, the main mechanism contributing to the promoted ablation performance for long-pulse laser IALE requires further verification. This requires the quantitative measurement of the morphologies of craters irradiated by lasers in various environments. Conventionally, measurement methods including

*Corresponding author (email: 1fu@mail.hust.edu.cn) histological methods, scanning electron microscopy, atomic force microscopy, X-ray computed tomography, and optical coherence tomography (OCT) have been performed to measure quantitatively the morphologies of craters induced by pulsed lasers [11]. The histological methods require the sample to be cut into serial sections and then be imaged by microscopy. This is a destructive process, and the possibility of damage during the cutting process cannot be excluded. In the case of scanning electron microscopy, samples need to be coated with a thin film of conducting material [12]. Atomic force microscopy has a limited range of scanning and is thus only suited to small samples with size of the order of hundreds of microns [13]. X-ray computed tomography is a non-contact and non-preprocessing method, but it requires a longer time to implement and has less resolution of approximately tens of microns [14]. In contrast, OCT is a potential tool for monitoring the variation in the geometrical shape of the crater with non-contact, high-speed, and nonpreprocessing advantages. OCT usually has resolution of 
approximately a dozen microns and a measurement range of up to several millimeters. Unfortunately, OCT has insufficient resolution of a couple of microns for the quantitative analysis of surface roughness and micro-structural variation, which are important in evaluating laser ablation performance. The imaging depth and lateral resolution of OCT are contrary.

In this work, we present an optical coherence microscopy (OCM) system that measures quantitatively the morphologies of laser-induced craters while having advantages over the conventional OCT system, and will clarify the ablation difference for long-pulse-laser ablation IALE and in air. As a result, the ablation mechanism may be greatly clarified.

A schematic diagram of the full-field OCM system used in this experiment is shown in Figure 1. The system employs a tungsten halogen light source with output power of $10 \mathrm{~mW}$. The light is collimated by an achromatic lens and split into reference and sample beams by a beam splitter that is coated for the visible wavelength range. Two neutraldensity filters are added to the reference beam and sample beam, respectively, to match the back scattering intensity and to compensate for dispersion. Light is then converged by a pair of $10 \times / 0.3$ numerical aperture (NA) objectives to a silver-coated mirror at the reference beam and the hard biological tissues at the sample beam. The hard tissues are put on a piezoelectric transducer stage (PI, E-710.3CD, P563.3CD) that moves vertically during axial scanning. The reflected light and back-scattering light of the two beams are combined by the same beam splitter to a tube lens and lens 2, and finally imaged on a two-dimensional camera (Silicon Imaging, SI-1300-M-CL). Lens 2 is used to adjust the magnification.

In imaging biological soft tissue, full-field OCM usually employs a waveband at 800 or $1310 \mathrm{~nm}$, which is within the

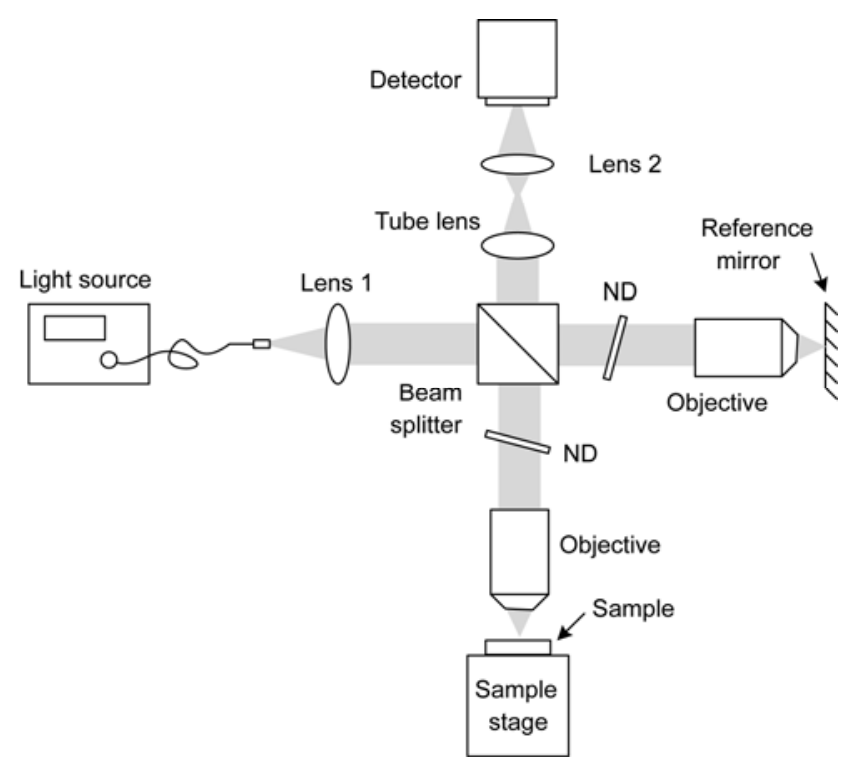

Figure 1 Schematic diagram of the full-field OCM system. imaging window for soft tissue. In our system, the visiblelight waveband is chosen because we only need to acquire the surface configuration of the hard tissue. Therefore, it is not necessary to consider the penetration depth into the hard tissue and the visible light facilitates the operation comparing with the infrared light. Because of the response of the optical elements and the camera, a 250-nm bandwidth (FWHM) centered at $650 \mathrm{~nm}$ is used for this system, corresponding to $\sim 0.8 \mu \mathrm{m}$ axial resolution theoretically. The axial resolution is measured using a mirror as a sample and scanning the piezoelectric transducer stage in the axial direction with a step size of $0.05 \mu \mathrm{m}$. At the same time, the mirror in the reference beam is kept static. The measured axial resolution is $1.1 \mu \mathrm{m}$ as shown in Figure 2(a). However, to reduce the imaging time, we choose the step size of the scanning as $2 \mu \mathrm{m}$, and the actual axial resolution is thus $2 \mu \mathrm{m}$ when measuring the craters in hard tissues. The camera is a progressive-scan digital camera, with $1280 \times 1024$ pixels and 5.2- $\mu \mathrm{m}$ pitch, and the frame rate is $\sim 25$ frames/s. Taking into account the scanning time and reconstruction time, $4 \mathrm{~s}$ is required to obtain an en-face image. Comprehensively considering the field of view, the NA of the objective, magnification, and pixel pitch, the final lateral resolution is $3 \mu \mathrm{m}$ as shown in Figure 2(b), which is obtained by measuring the sharp edge of the 1951 USAF glass slide resolution target. Adjusting the magnification by moving the position of lens 2 , the field of view of the system can reach $\sim 1.1 \mathrm{~mm}$. For this measurement, the field of view is $900 \mu \mathrm{m} \times 720 \mu \mathrm{m}$ as shown in Figure 2(c). The 1951 USAF glass slide resolution target was also imaged by the system, with the elements of groups 5, 6, and 7, and part of group 4 being clearly imaged (Figure 2(d)).

Figure 3 shows scanning images from $X Z, Y Z$, and $X Y Z$ axial directions for urinary calculus (calcium oxalate monohydrate (COM) and uric acid (UC)) craters irradiated in air and in water by a holmium laser $(300 \mu \mathrm{s}, 300 \mathrm{~mJ}, 1 \mathrm{~Hz})$ transmitted in $600 \mu \mathrm{m}$ low- $\mathrm{OH}^{-}$quartz fiber (length of $2 \mathrm{~m}$, NA of 0.22 ). The fiber is placed perpendicularly in contact with the calculus surfaces in our experiments and the calculus surfaces are at least $1 \mathrm{~cm}$ under the water surface. The heights and widths of ablation craters are labeled in the corresponding images. Cross sectional profiles aligned with the deepest positions of the craters are displayed. The experimental results indicate the following. (1) The depth of a crater increases nonlinearly with the number of pulses either in water or in air. The augment speed of crater heights in water is higher than that in air. (2) The surface diameters (widths) of the craters are smaller than the diameter of the fiber core used except in the case of multiple-pulse irradiation owing to the relative position shifting between the fiber end and tissue surface induced by the recoil force. The craters induced in air have smaller crater depths and widths than those induced in water under the same condition. Ablation in air produces relatively rough and irregular contours, and some protrusions along the rims of craters (see red 

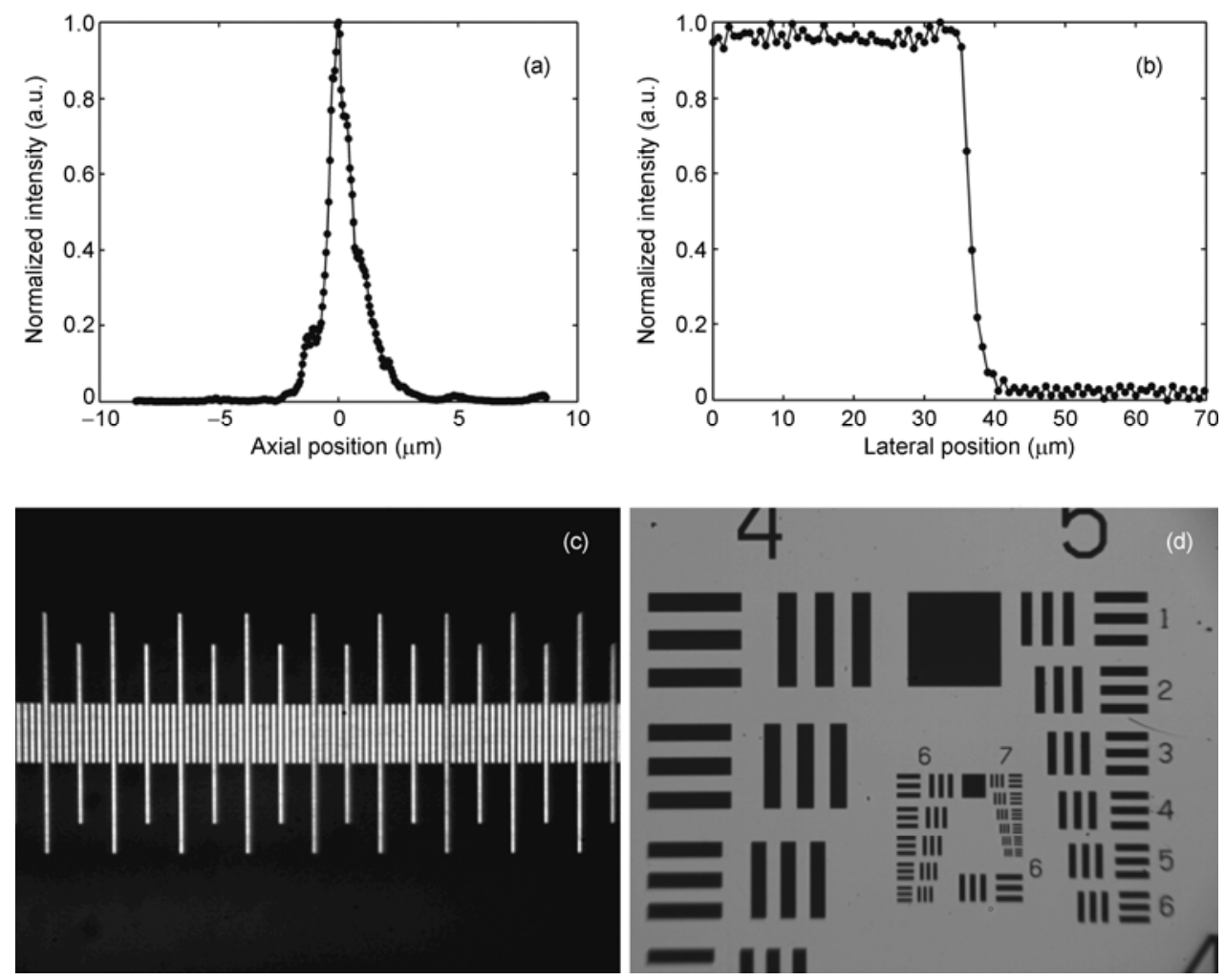

Figure 2 (a) Measured axial resolution of $1.1 \mu \mathrm{m}$; (b) measured lateral resolution of $3 \mu \mathrm{m}$; (c) field of view of the system of $900 \mu \mathrm{m}$ in the horizontal and $720 \mu \mathrm{m}$ in the vertical; (d) imaging of the 1951 USAF glass slide resolution target.

arrows) or at the bases of craters. Altshuler et al. [15] declared that solid particles of ablated material are accelerated against the walls of a crater, resulting in a polishing effect that removes debris and any protruding sharp edges. However, the obvious morphology difference in ablation craters in air and in water shows that Altshluler's statement is not absolutely correct. This implies that the role of the water layer needs to be reconsidered. We suggest that the mechanism of water augmentation is that the forces resulting from explosive vaporization are imparted on the crater surface by recoiling water during cavitation and remove any undesirable surface protrusions or asperities in the ablation crater, which are likely to inhibit efficient ablation of subsequent laser pulses leading to excessive heat accumulation. This is in good agreement with the opinion of Staninec [16]. Furthermore, the recoil stress produced by both vaporization and material ejection through explosion and confined boiling can induce a secondary material expulsion that decreases the shielding effect and greatly increases the ablation efficiency [6]. Additionally, because a holmium laser pulse consists of multiple spikes (with nanosecond width), each laser spike can satisfy the stress confinement condition and induce a bipolar acoustic wave [17]. A negative wave of a bipolar wave can decrease the ablation threshold of tissue because tissue is more susceptible to damage from tensile stress and shear stress than to damage from compressive stress [18]. (3) For the same laser parameters, the perfor- mance of ablation in air and that in water for single laser pulse are comparable, but the latter is slightly better than the former. This result conflicts with that for short-pulse laser ablation IALE [1,2]. (4) With the number of laser pulses increasing, ablation in water outperforms that in air, as evidenced by the larger ablation volume, deeper crater height, and more lubricious crater rim. Crater heights induced by 6 and 10 pulses in air have no obvious difference, whereas crater heights for 10 pulses are greater than those for 5 pulses in water. The main reasons for this finding are that there is a greater increase in energy conversion efficiency and a stronger vaporization effect contributing to ablation. The force resulting from the explosive vaporization of water, but not the shock waves resulting from collapses of cavitation bubbles, strongly promotes ablation.

Full-field OCM is similar to OCT in that it employs white-light interferometry to obtain the three-dimensional structure of the tissue; however, it has much higher resolution of $\sim 1 \mu \mathrm{m}$ and does not require optical scanning of the sample surface [19]. The three-dimensional structure is obtained by reconstructing the en-face images. A tungsten halogen light source is used in the full-field OCM system as an incoherent light source with very wide bandwidth of hundreds of microns leading to ultrahigh axial resolution to the submicron level. The lateral resolution is limited by the NA of the objective, which is the same as in normal microscopy. A dynamic focus is used to keep the high lateral 


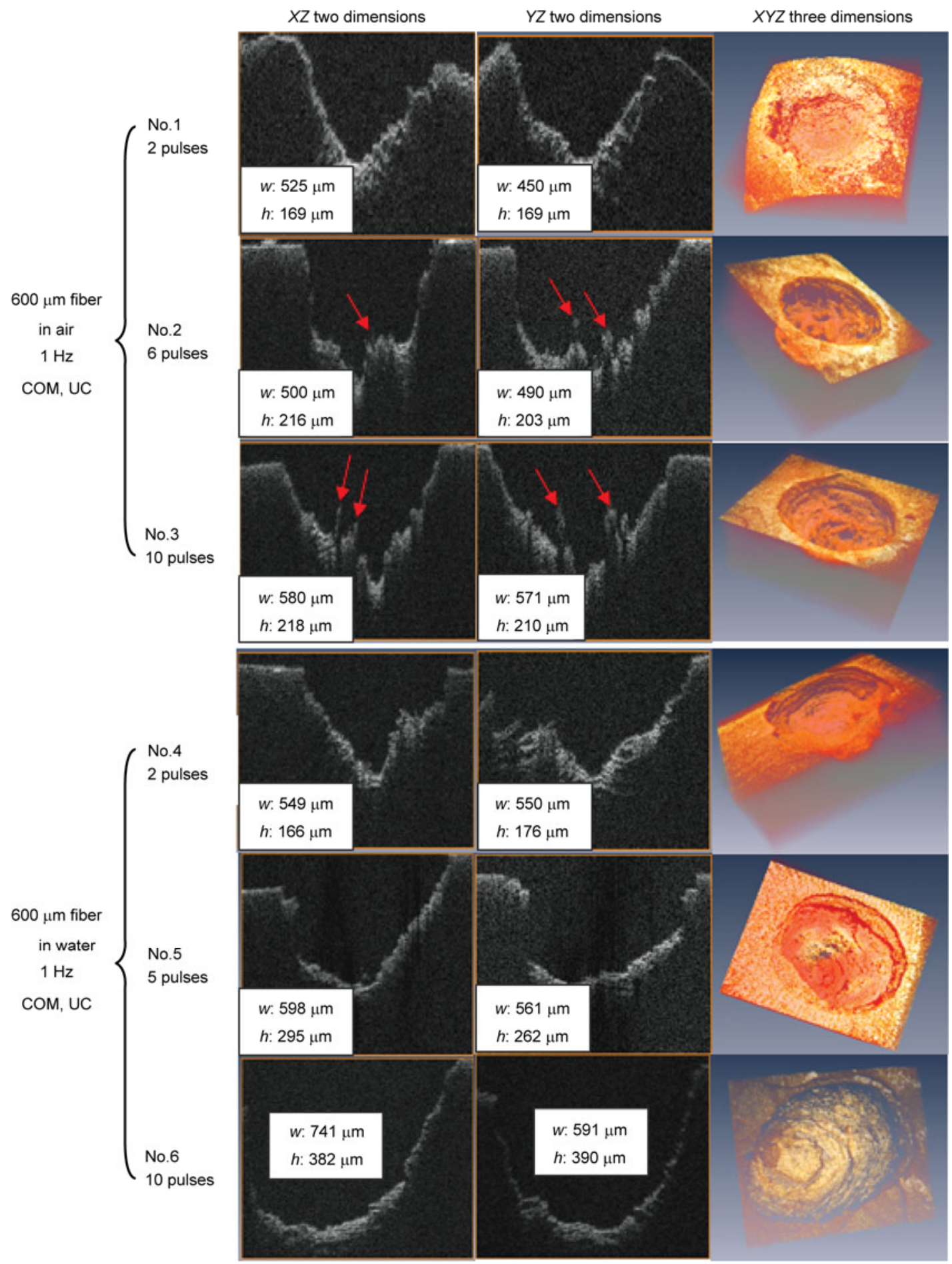

Figure 3 Cross sectional topography of holmium laser-induced $(300 \mu \mathrm{m}, 300 \mathrm{~mJ}, 1 \mathrm{~Hz})$ craters acquired with the OCM system in air and in water. The $600-\mu \mathrm{m}$ fiber is perpendicularly placed in contact with urinary calculus surfaces. $w$ : width; $h$ : height.

resolution during the depth scanning, and the range of the imaging depth is only determined by the mechanical scanner and working distance of the objective. The OCM is commonly applied to the examination of ocular tissues [20], cells [21], and semiconductors [22]. However, the applications of full-field OCM for the characterization of laser- induced craters in hard tissue have not yet been reported. Our measurements for calculus craters employing an OCM system are one order of magnitude higher than those employing an OCT system [3,4], which will help us evaluate the difference in ablation performance during laser-tissue interaction in water and in air by analyzing the shape and 
volume of craters accurately.

We presented an OCM system with obvious non-contact, non-destructive, non-preprocessing and high-resolution $(\sim 1$ $\mu \mathrm{m})$ advantages, with which it is more feasible to measure quantitatively the three-dimensional structure and cross sectional profiles of pulsed lasers-induced craters in hard biological tissues in various environments, compared with using conventional measurement means. As a result, the dynamic characteristics of ablation performance differences in water and in air were greatly clarified. Our research results will be valuable to surgeons in that they will aid safe, efficient, and low-cost surgery in real medical applications.

This work was supported by the National Natural Science Foundation of China (61008054), the Program for New Century Excellent Talents in University (NCET-08-0216), the Special Fund for Basic Scientific Research of Central Colleges, China University of Geosciences (Wuhan) (CUG090112, CUG110408) and the Program for Wuhan City Youth Chenguang Technology.

1 Daminelli G, Krüger J, Kautek W. Thin Solid Films, 2004, 467: 334-341

2 Zhu S, Lu Y F, Hong M H. Appl Phys Lett, 2001, 79: 1396-1398

3 Lv T, Xiao Q, Xia D Q, et al. J Biomed Opt, 2010, 15: 048002

4 Kang H W, Oh J, Welch A J. Phys Med Biol, 2008, 53: 3381-3390

5 Fried D, Ashouri N, Breunig T, et al. Lasers Surg Med, 2002, 31.
186-193

6 Vogel A, Venugopalan V. Chem Rev, 2003, 103: 577-644

7 Fanton G S, Dillingham M F. Semin Orthop, 1992, 7: 102-116

8 Ith M, Pratisto H, Staubli H U, et al. Sports Exercise Injury, 1996, 2: 207-209

9 Cross F W, Al-Dhahir R K, Dyer P E. J Appl Phys, 1988, 64: 2194-2201

10 Lee H, Kang H W, Teichman J M H, et al. Lasers Surg Med, 2006, 38: $39-51$

11 Diaci J. J Laser Health Acad, 2008, 2008: 2

12 Kima Y, Choia E S, Kwaka W, et al. Opt Laser Tech, 2008, 40: 625-631

13 O'Mahony C, Hill M, Brunet M, et al. Meas Sci Tech, 2003, 14: 1807-1814

14 Mercer C E, Anderson P, Davis G. Int Congress Ser, 2003, 1248: 131-134

15 Altshuler G B, Belikov A V, Sinelnik Y A. Lasers Surg Med, 2001, 28: 435-444

16 Staninec M, Xie J, Le C Q, et al. Lasers Surg Med, 2003, 33: 264-269

17 Frenz M, Pratisto H, Konz F, et al. IEEE J Quant Electron, 1996, 32: 2025-2035

18 Albagli D, Dark M, Perelman L T, et al. Opt Lett, 1994, 19: 1684-1686

19 Chen Y, Huang S W, Aguirre A D, et al. Opt Lett, 2007, 32: 1971-1973

20 Akiba M, Maeda N, Yumikake K, et al. J Biomed Opt, 2007, 12: 041202

21 Zhou C, Wang Y, Aguirre A D, et al. J Biomed Opt, 2010, 15: 016001

22 Stifter D. Appl Phys B, 2007, 88: 337-357

Open Access This article is distributed under the terms of the Creative Commons Attribution License which permits any use, distribution, and reproduction in any medium, provided the original author(s) and source are credited. 